\title{
Pädiatrische Versorgungskonzepte in Europa
}

M.W. Weber ${ }^{1}$, S. Backhaus ${ }^{1}$, O. Chukwujama ${ }^{2}$, F. Fenski ${ }^{3}$, C. Henking ${ }^{4}$, L. Schatte ${ }^{5}$, A. Aleman $\operatorname{Diaz}^{6}$

${ }^{1}$ WHO Regionalbüro für Europa, Kopenhagen, Dänemark

${ }^{2}$ Ludwig-Maximilians-Universität, München, Deutschland

${ }^{3}$ Freie Universität Berlin, Deutschland

${ }^{4}$ London School of Economics and Political Science, London, Großbritannien

${ }^{5}$ King's College London, London, Großbritannien

${ }^{6}$ University of St. Andrews, Großbritannien

$\underline{\text { Korrespondenzadresse: }}$

PD Dr. M. Weber

WHO Regionalbüro für Europa

Marmorvej 51, 2100 Kopenhagen, Dänemark

weberm@who.int

Tel. +4530311617

MWW ist Mitarbeiter der Weltgesundheitsorganisation. Die ausgedrückte Meinung ist seine eigene und entspricht nicht notwendigerweise der Politik und den Positionen der WHO. 
Foto

\section{Interessenkonflikt:}

M.W. Weber, S. Backhaus, O. Chukwujama, F. Fenski, C. Henking, L. Schatte und A. Aleman Diax geben an, dass kein Interessenkonflikt besteht. Dieser Beitrag beinhaltet keine von den Autoren durchgeführten Studien an Menschen oder Tieren.

\section{Danksagung.}

Die Autoren danken Prof. Mitch Blair und Prof. Michael Rigby, Imperial College, London, für die Interpretation von MOCHA Daten, und Aigul Kuttumuratova für Daten über die Versorgungssysteme in Osteuropa und Zentralasien.

\section{Trailer}

Die europäische Kinder- und Jugendstrategie der Weltgesundheitsorganisation dient Ländern als Anleitung zur Implementierung von Maßnahmen in diesem Bereich. Die Basiserhebung der WHO identifizierte Versorgungslücken, die angegangen werden können. Der Artikel stellt Lücken an essentiellen Medikamentenlisten und Darreichungsformen für Kinder, Kinderpsychiatern und in Aspekten sexueller und reproduktiver Gesundheit in europäischen Ländern dar. Pädiater sollten über diese Datenquellen und internationale Richtlinien Bescheid wissen.

\section{Zusammenfassung}

Zur Förderung der Kindergesundheit in Europa hat die Weltgesundheitsorganisation (WHO) die Kinder- und Jugendstrategie 2015-2020 erarbeitet, die von allen Mitgliedstaaten angenommen wurde und von ihnen umgesetzt werden soll. Um die Implementierung auf Länderebene zu dokumentieren, führte das europäische Regionalbüro 2016 eine Befragung durch, an der 48 von 53 Ländern teilnahmen. Dieser Artikel stellt ausgewählte Ergebnisse dar; alle Aspekte werden in einem WHO Report und auf der WHO Webseite verfügbar sein. 
Bei der Primärversorgung von Kindern und Jugendlichen verfügt Europa über viele Versorgungsmodelle. Die meisten Länder verwenden ein System, in dem Kinder von Pädiatern und von Allgemeinärzten behandelt werden. Bei der Medikamentenversorgung haben viele Länder keine pädiatrische essenzielle Medikamentenliste sowie nicht alle der häufigen Medikamente in pädiatrischen Darreichungsformen - auch Deutschland nicht. Wesentliche Daten zur psychischen Gesundheit von Jugendlichen kommen aus der Studie zum Gesundheitsverhalten von Kindern im Schulalter (HBSC), die zu beispielhaften nationalen Aktionen geführt hat. Zugang zu sexuellen und reproduktiven Gesundheitsdiensten ist in der europäischen Region vielerorts ein Problem, so ist ein legaler Schwangerschaftsabbruch ohne elterliche Zustimmung für Jugendliche unter 18 Jahren nur in Hälfte der befragten Länder möglich.

Der Artikel stellt die deutschen Verhältnisse in Bezug zu denen in anderen europäischen Ländern, und gibt Hinweise auf die wesentlichen Indikatoren der Kinder- und Jugendgesundheit in Europa sowie die Quellen, wo diese nachverfolgt werden können. Dies hilft im politischen Dialog, auch der Kinderärzte als Anwälte der Kinder, um relevante Empfehlungen zur Verbesserung der Kinder- und Jugendgesundheit abzugeben.

\title{
Schlüsselwörter
}

Kindergesundheit, Gesundheitssystem, Primärversorgung, Health Behaviours in School-aged Children, Weltgesundheitsorganisation

\begin{abstract}
To promote children's health in Europe, the World Health Organization (WHO) developed the European child and adolescent health strategy 2015-2020, which is supported and will be implemented by all Member States (MS). In order to measure the implementation of the strategy at country level, the WHO Regional Office carried out a survey in 2016, involving 48 out of 53
\end{abstract}


countries (91\%). Findings from the survey will be available in a forthcoming WHO report and on the WHO website. This article presents selected results about health systems, mental and sexual and reproductive health.

The primary care of children and adolescents in Europe illustrates a variety of care models, yet

the majority of MS incorporate a system in which children are treated by both paediatricians and general practitioners. The survey also shows that many countries, including Germany, do not have paediatric essential drug lists or paediatric formulations of all essential drugs. Key data on mental health of adolescents derived from the Health Behaviour in School-aged Children (HBSC) study, included in WHO Europe monitoring profiles of child health, has led to exemplary national actions. Access to sexual and reproductive health services continues to be a problem in many countries. A legal abortion without parental consent for adolescents under the age of 18 is only possible in less than half $(48 \%)$ of the countries surveyed.

This article compares the German case with that of other European countries and provides policy directions for the main childhood and youth health indicators in Europe as well as where they can be tracked. The ultimate aim is to support political dialogue, recognizing paediatricians as advocates for children, to make relevant recommendations for improving child and adolescent health.

\section{Keywords}

child health, health systems, primary care, Health Behaviours in School-aged Children, World Health Organization 


\section{Einleitung}

Europa ist vielfältig, und vielfältig sind auch die Versorgungssysteme für Kinder. Alle europäischen Staaten haben sich dem Kindeswohl als allgemeinen Maßstab verpflichtet, indem sie die UN Kinderrechtskonvention unterschrieben haben [17]. Diese hat im Artikel 24 eine Verpflichtung der Staaten zur Förderung der Kindergesundheit festgeschrieben. Um hierzu eine praktische Anleitung zu geben, hat das europäische Regionalbüro der Weltgesundheitsorganisation (WHO) die Kinder- und Jugendgesundheitsstrategie 2015-2020 erarbeitet, die von allen 53 Mitgliedsstaaten der Region angenommen wurde [22]. Sie gibt Richtlinien vor, an denen sich die Staaten orientieren und sie in ihre Gesetze und Verordnungen umsetzen sollen. Außerdem stellt sie Bereiche fest, in denen Daten zur Überwachung und Evaluierung erhoben werden sollen. Der Artikel gibt eine Übersicht, wie diese Ziele von den europäischen Staaten umgesetzt werden und beschreibt Beispiele der europaweiten Datenerhebung der WHO.

\section{Die Kinder- und Jugendstrategie der WHO für Europa}

Die Kinder- und Jugendstrategie 2015-2020 verfolgt das Ziel, es Kindern und Jugendlichen im europäischen Raum der WHO zu ermöglichen, ihr volles Potenzial zur Gesundheit, Entwicklung und zum Wohlergehen auszuschöpfen; sowie ihre Belastung durch vermeidbare Krankheiten und damit die Sterblichkeit zu verringern (Textbox1).

\section{Textbox 1 hier}

Zwar findet man im europäischen Raum Länder mit der niedrigsten Säuglings-und Kindersterblichkeit der Welt, jedoch sind die Unterschiede zwischen den Ländern gravierend (Abb. 1). So ist die Sterblichkeit in einigen Ländern 25-mal höher als in Ländern mit geringer Kindersterblichkeit.

\section{Abbildung 1 hier}




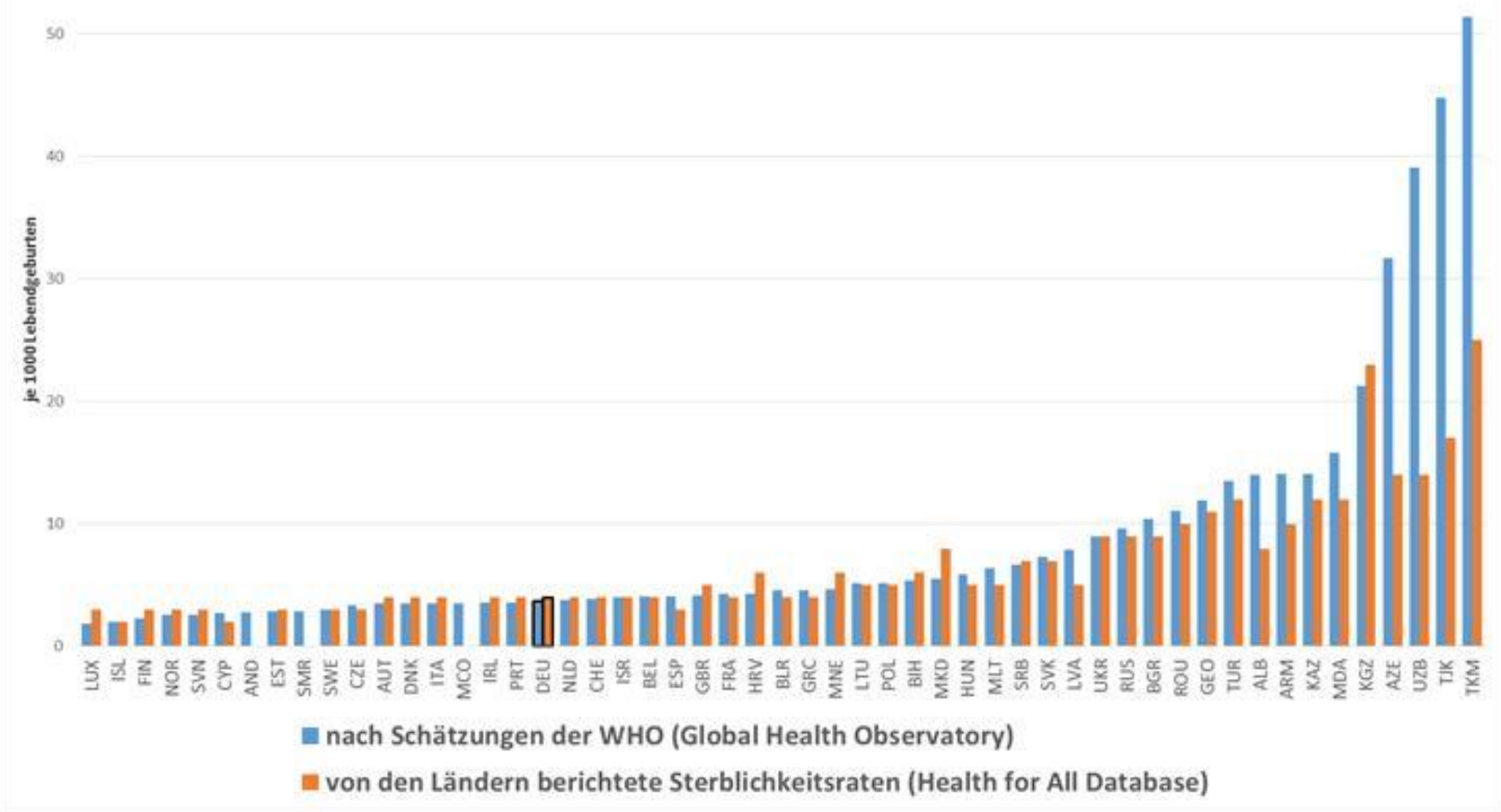

Als weiteres Ziel der Strategie fordert die WHO die einzelnen Länder dazu auf, ihre eigenen Ziele zu setzen, um spezifische Bedürfnisse zu erfüllen.

\section{Die Basiserhebung zur Kinder- und Jugendstrategie in den europäischen Ländern}

Um die Fortschritte bei der Umsetzung der Strategie messbar zu machen, wurde 2016 eine Basiserhebung in den Mitgliedstaaten durchgeführt, die den Stand der Kinder- und Jugendgesundheit in der europäischen Region erfassen sollte. Diese Erhebung ergänzt die bereits veröffentlichten Daten, die die WHO in Länderprofilen auf ihrer Webseite allgemein verfügbar macht, und die auf früheren Studien und regelmäßig gesammelten Indikatoren beruhen (Informationen unter https://gateway.euro.who.int/en/). Der Fragebogen der Basiserhebung wurde mit Hilfe eines Online-Umfrage-Tools (surveygizmo) erstellt und im August 2016 an die Gesundheitsministerien der 53 Mitgliedstaaten verschickt. Die Teilnehmer wurden gebeten, gegebenenfalls Experten für spezifische Themenbereiche zu konsultieren. Der Fragebogen konnte auf Englisch oder Russisch ausgefüllt werden. Nach der ersten Bitte zur Teilnahme wurden Erinnerungen an die Länder mit ausstehenden Antworten verschickt. Bei Abschluss der Umfrage am 31.03.2017 ergab das Antworten aus 48 Ländern. Fünf Länder starteten die Befragung trotz mehrerer Aufforderungen nicht. 
Die Fragen deckten verschiedene Aspekte der Strategie für Kinder- und Jugendgesundheit ab. Von den insgesamt 82 Fragen untersuchten $68 \%$, ob eine bestimmte politische Richtlinie, die von den WHO-Richtlinien und der Strategie empfohlen wird, im jeweiligen Land implementiert ist (z.B. Sammeln Sie systematische Informationen zum Alkoholkonsum von Müttern?). Die Antworten wurden zuerst deskriptiv analysiert und anhand von Karten visualisiert, um die Verteilung der Antworten zu politischen Richtlinien und numerischen Indikatoren in der Region darzustellen (Beispielkarte siehe Abbildung 2). Analysen wurden außerdem bezüglich der folgenden politisch-geografische Gruppierungen durchgeführt: die EU 15, welche sich aus den ältesten Mitgliedern der EU zusammensetzt (Belgien, Dänemark, Deutschland, Finnland, Frankreich, Griechenland, Großbritannien, Irland, Italien, Luxemburg, Niederlande, Österreich, Portugal, Spanien, Schweden), die EU 13 mit seit 2004 hinzugekommenen Mitgliedsstaaten (Bulgarien, Kroatien, Zypern, Tschechische Republik, Estland, Ungarn, Lettland, Litauen, Malta, Polen, Romania, die Slowakei und Slowenien), die Länder des Commonwealth of Independent States; CIS (Azerbaijan, Armenien, Belarus, Kazakhstan, Kirgisistan, Moldawien, Russland, Tadschikistan, Turkmenistan, Usbekistan, Ukraine) sowie die Mitglieder des südosteuropäischen Gesundheitsnetzwerkes (SEEHN: Albanien, Bosnien und Herzegowina, Bulgarien, die ehemalige jugoslawische Republik Mazedonien, Kroatien, Montenegro, Republik Moldawien, Rumänien und Serbien).

Davon ausgehend wurden die Ergebnisse schließlich mit anderen zentralen Indikatoren (u.a. Sterblichkeitsraten und kinderärztliche Versorgung) sowie Daten über die Verhaltensebene der Kinder- und Jugendgesundheit in Verbindung gebracht. Dieser Artikel stellt ausgewählte Ergebnisse aus den Bereichen Arzneimittelverfügbarkeit, psychische Gesundheit sowie der sexuellen und reproduktiven Gesundheit dar.

Neben den Ergebnissen der Basisbefragung wurden Modelle der Grundversorgung von Kindern durch die Kombination unterschiedlicher Datenquellen analysiert: die Models of Child Health Appraised (MOCHA) Studie pädiatrischer Grundversorgung in 30 europäischen Ländern [12], die Europäische Beobachtungsstelle für Gesundheitssysteme und Gesundheitspolitik, Expertenmeinungen von nationalen Professional Officers der WHO in den Länderbüros und Artikeln eines Supplements des Journal of Pediatrics [1-3,5,6,10,13-15].

\section{Abbildung 2 hier}




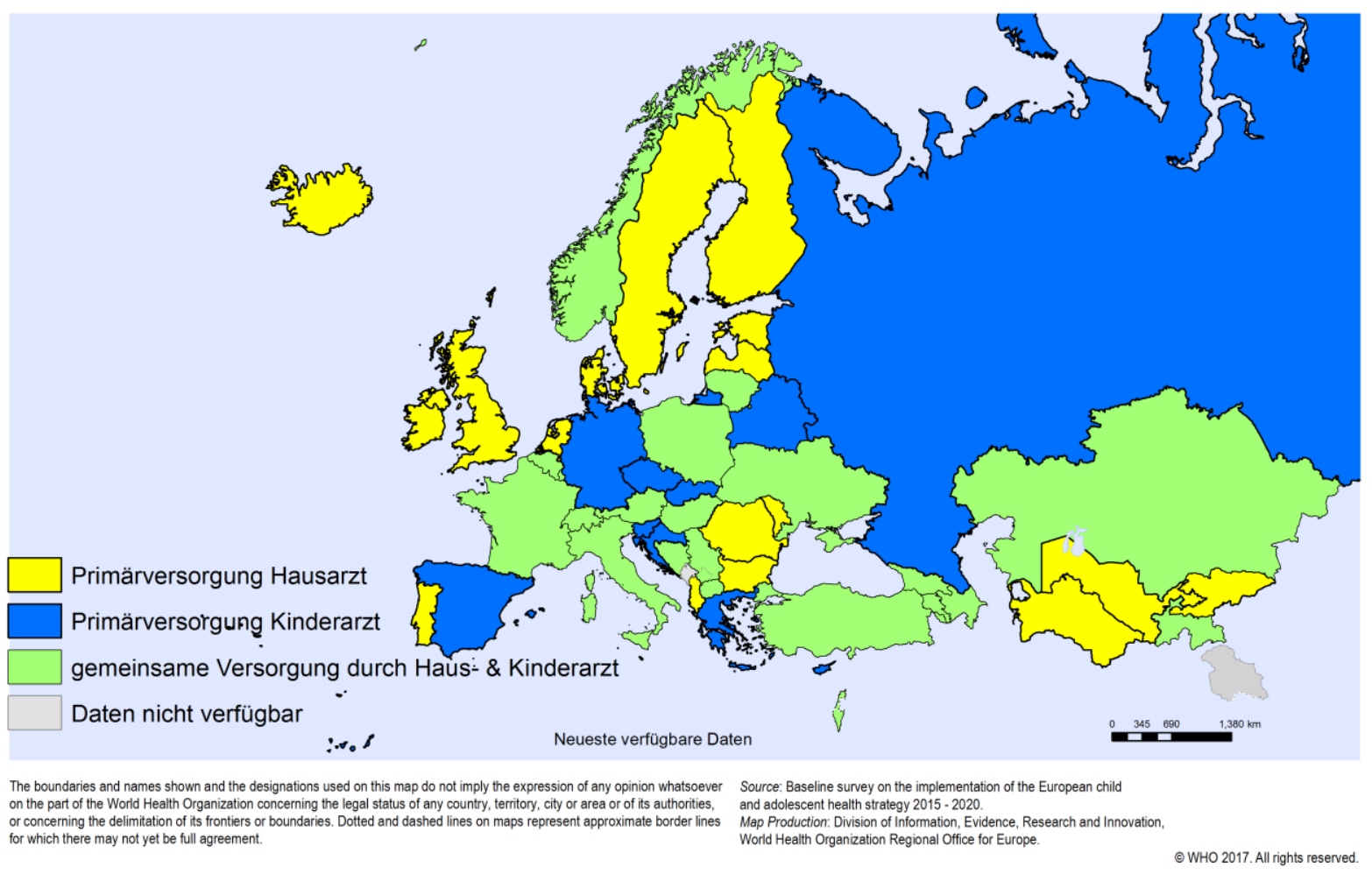

\section{Modelle der pädiatrischen Primärversorgung in Europa}

Dieser Abschnitt vergleicht die medizinische Grundversorgung von Kindern und Jugendlichen zwischen den 53 Mitgliedstaaten der europäischen Region der WHO. Unterschieden wird hierbei zwischen der Primärversorgung durch (A) vorwiegend Kinderärzte, (B) überwiegend Hausärzte und (C) gemeinsame Versorgung durch Kinder- und Hausärzte; basierend auf der Spezialisierung des für den Erstkontakt zuständigen Arztes. Diese Herangehensweise geht aus einem von MOCHA [12] entwickelten Ansatz hervor: In Model A erfolgt die Versorgung eines Kindes aufgrund kurativer, präventiver oder medizinischer Interventionen gewöhnlich von einem Mediziner mit postgradualer Spezialisierung in Pädiatrie. Hingegen ist in Modell B der Allgemeinmediziner der erste Ansprechpartner für medizinische Konsultationen bei Kindern. In Modell C spielen sowohl Kinderärzte als auch Hausärzte eine wichtige Rolle in der Primärversorgung von Kindern. 


\section{Einordnung der europäischen Länder in die drei Versorgungsmodelle}

Knapp ein Fünftel (19\%) der Mitgliedsstaaten der europäischen Region wurden Model A (Primärversorgung durch Kinderarzt) zugeordnet, wie zum Beispiel Belarus, Zypern, Tschechische Republik, Deutschland, Spanien, Griechenland, Slowenien und Russland. Die Länder, die als Model B identifiziert wurden (36\% der Mitgliedstaaten) waren u.a. Albanien, Dänemark, Island, Kirgisistan, Niederlande, Vereinigtes Königreich und Usbekistan. Die übrigen 45\% der Staaten gehören dem Modell C (gemeinsame Versorgung) an. Diese Angaben sind deskriptive Beschreibungen. Vor- und Nachteile der Modelle werden momentan durch das MOCHA-Projekt der EU untersucht. Erste Ergebnisse sind 2018 zu erwarten.

Gemeinde-Kinderkrankenschwestern spielen in Dänemark, Großbritannien, Frankreich und Finnland eine große Rolle in der Primärversorgung der Kinder. Besuche solcher Krankenschwestern werden Familien mit Neugeborenen für die ersten Wochen nach der Geburt und während der Schwangerschaft in Dänemark angeboten [11]. Diese Form von Erstversorgung existiert ebenfalls in Portugal und Spanien, wo die Krankenschwestern die Mütter zusätzlich in Bezug auf Stillen, Impfungen und medizinischen Untersuchungen beraten [9]. In Großbritannien führen sogenannte Gesundheitsbesucher (geschulte Krankenschwestern und Hebammen) regelmäßig Hausbesuche bei Familien mit Neugeborenen durch. Zusätzlich übernehmen Schulkrankenschwestern die Versorgung von Kindern im Schulalter [19]. Krankenschwestern und Hebammen aus Gesundheitszentren in Finnland fungieren als Weichensteller zu Allgemeinärzten, verfügen aber über weniger Rechte in Bezug auf Medikamentenverschreibung bei Kindern unter 12 Jahren.

\section{Nationale essentielle Medikamentenlisten und Darreichungsformen für Kinder}

Im Jahre 2007 wurde die Resolution „Better Medicines for Children“ von der Weltgesundheitsversammlung verabschiedet [21]. Ziel dieser Resolution war es, Forschung und Entwicklung von Medikamenten für Kinder voranzubringen. Eine Errungenschaft dieser Resolution stellt die Model List of Essential Medicines for children (EMLc) dar; eine Medikamentenliste, die als Orientierung zur Entwicklung einer nationalen essentiellen Medikamentenliste dient. Ergebnis dieser sollte eine Auswahl an Medikamenten auf aktuellem Stand, die Qualitätssicherung der Arzneimittel, sowie die ständige Weiterentwicklung von 
Arzneien, Notfallmedikationen und Pharmazeutika, die veränderten Resistenzmustern begegnen, sein.

Unsere Erhebung zeigt, dass viele Länder im europäischen Raum eine nationale Liste bisher nicht umgesetzt haben. Nur knapp ein Drittel der Länder (15 von 48) gab an, über eine pädiatrische essentielle Medikamentenliste zu verfügen. Von den Ländern, die mit „Ja“ antworteten, machten $87 \%$ die Liste für die Öffentlichkeit zugänglich. Deutschland gehört zu den 25 Ländern, die über keine pädiatrische essentielle Medikamentenliste verfügen (Abb.3).

\section{Pädiatrische Darreichungsformen in Deutschland}

Weiterhin wurden die Länder befragt, ob pädiatrische Darreichungsformen für alle wesentlichen Medikamente verfügbar sind. Von den 48 Ländern bejahten diese Frage lediglich 17. Es mangelt vor allem in vielen westeuropäischen Ländern an der essentiellen Medikamentenliste und Darreichungsformen für Kinder. Deutschland stellt dabei keine Ausnahme dar; osteuropäische Länder haben dies stärker auf nationaler Ebene umgesetzt.

Aus obengenannten Gründen hält die WHO eine national angepasste Liste für bedeutsam. Ungenügende pädiatrisch angepasste Darreichungsformen können auch durch fehlendes Interesse seitens pharmazeutischer Unternehmen begründet sein, da der Markt für Kinder und Ethikrichtlinien für Studien Hürden darstellen. Nur Arzneimittel mit pädiatrischer Zulassung ermöglichen jedoch eine sichere und exakte Dosierung. Die jeweiligen Berufsverbände spielen eine wesentliche Rolle in diesem Dialog als Fürsprecher der Kinderinteressen.

\section{Dokumentation des Verschreibungsverhaltens}

Ein weiterer Qualitätsindikator ist das Sammeln systematischer Informationen über die Anzahl der Arzneimittelverordnungen an Kinder und Jugendliche unter 18 Jahren. Knapp die Hälfte der befragten Länder (45\%) bejahte, dass sie dies tun. Wurden die Länder allerdings nach konkreten Zahlen der Verschreibung von Pharmaka zur Behandlung psychischer Störungen und Verhaltensauffälligkeiten gefragt, konnten nur sieben Länder Zahlen nennen. Deutschland berichtete, dass 65700000 Tagesdosen dieser Medikamente an Kinder und Jugendliche im Jahr 2014 verordnet wurde.

\section{Abbildung 3 hier}




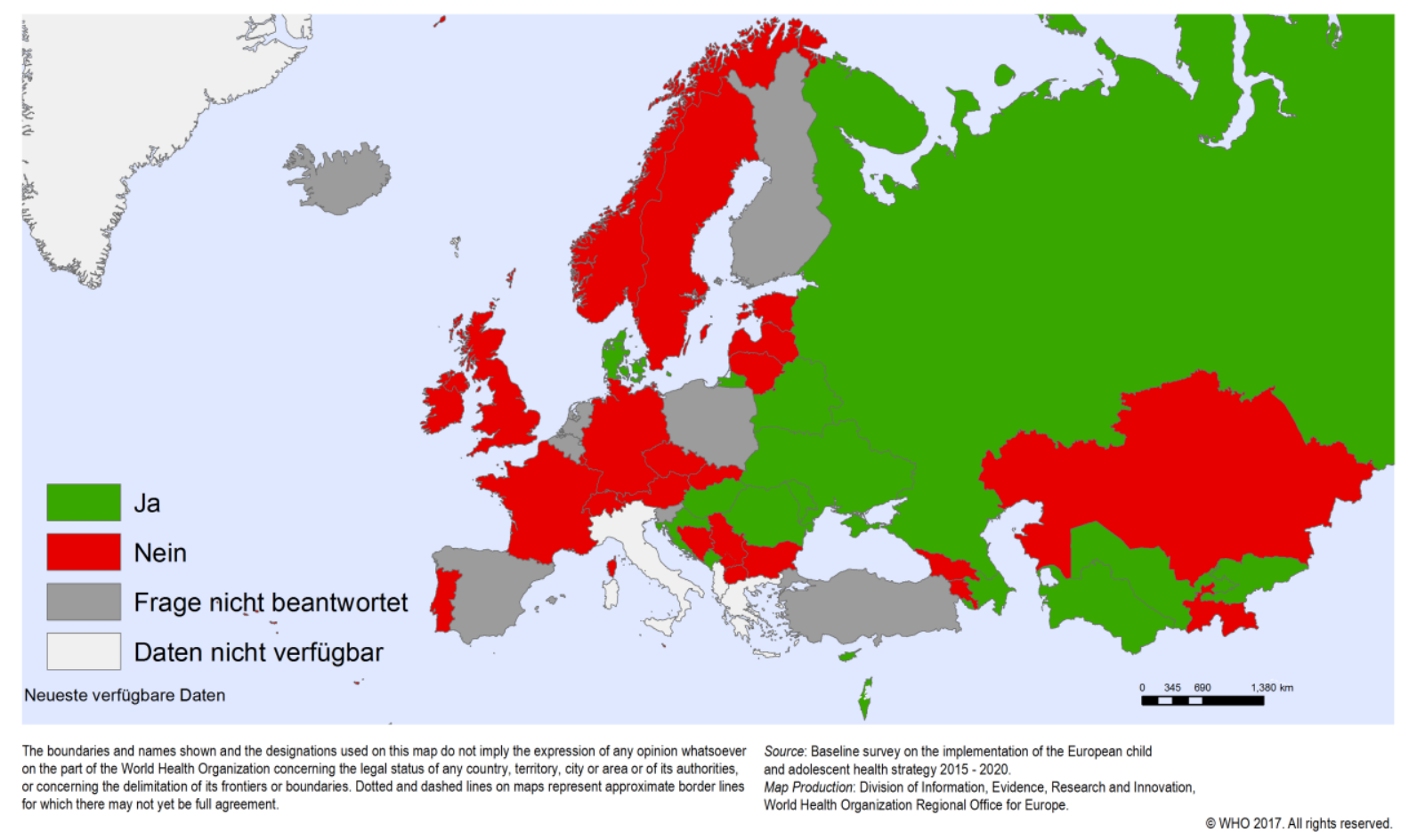

\section{Psychische Gesundheit}

Psychische Erkrankungen machen fast 20\% der Krankheitslast in der Europäischen Region der WHO aus. Dabei hat die Hälfte aller psychischen Krankheiten ihren Beginn vor dem 14. Lebensjahr [7]. Sie belasten nicht nur die betroffenen Menschen, sondern haben auch Auswirkungen auf das Leben der Betreuungspersonen und die Produktivität der Gesellschaft insgesamt. Psychische Gesundheitsprobleme haben Einfluss auf die soziale, intellektuelle und emotionale Entwicklung von Kindern und Jugendlichen und stehen im Zusammenhang mit psychischen Störungen im Erwachsenenalter [4].

\section{Gesundheitsverhalten von Kindern im schulpflichtigen Alter}

Die Health Behaviour in School-aged Children (HBSC) Studie ist eine Kollaboration des WHO Regionalbüros, an der 47 Länder und Regionen in Europa sowie Nordamerikas beteiligt sind [16]. In der alle vier Jahre durchgeführten Erhebung werden Mädchen und Jungen im Alter von 11, 13 und 15 Jahren nach ihrer Gesundheit und ihrem Wohlbefinden, ihren sozialen Umfeldern und 
ihrem Gesundheitsverhalten befragt. Als Maß für das psychische Wohlbefinden der Jugendlichen dienen subjektive gesundheitliche Variablen, wie selbsteingeschätzte Gesundheit, Lebenszufriedenheit und multiple Gesundheitsbeschwerden. Ergebnisse der Erhebung 2013/2014 zeigten, dass sich das psychische Wohlbefinden im Laufe der Pubertät verschlechtert. 15-Jährige berichteten signifikant häufiger von multiplen Gesundheitsbeschwerden (Abbildung 4) und von einer geringeren Lebenszufriedenheit betroffen $\mathrm{zu}$ sein als 11-Jährige. Klare Geschlechtsunterschiede zeigten sich über alle Altersstufen hinweg: Mädchen schätzten ihre Gesundheit schlechter ein, gaben eine geringe Lebenszufriedenheit an und bekundeten häufigere Gesundheitsbeschwerden. Zudem scheint das Wohlstandsniveau der Familie einen wichtigen Einfluss auf die psychische Gesundheit der Jugendlichen zu haben. Junge Menschen aus weniger wohlhabenden Haushalten gaben durchgängig eine niedrigere Lebenszufriedenheit, häufigere Gesundheitsbeschwerden und häufiger mittlere oder unzureichende Gesundheit an. Die Erhebung wies zudem auf substanzielle Unterschiede in Bezug auf die Prävalenz subjektiver psychischer Gesundheit zwischen den Ländern hin.

\section{Abbildung 4 hier}

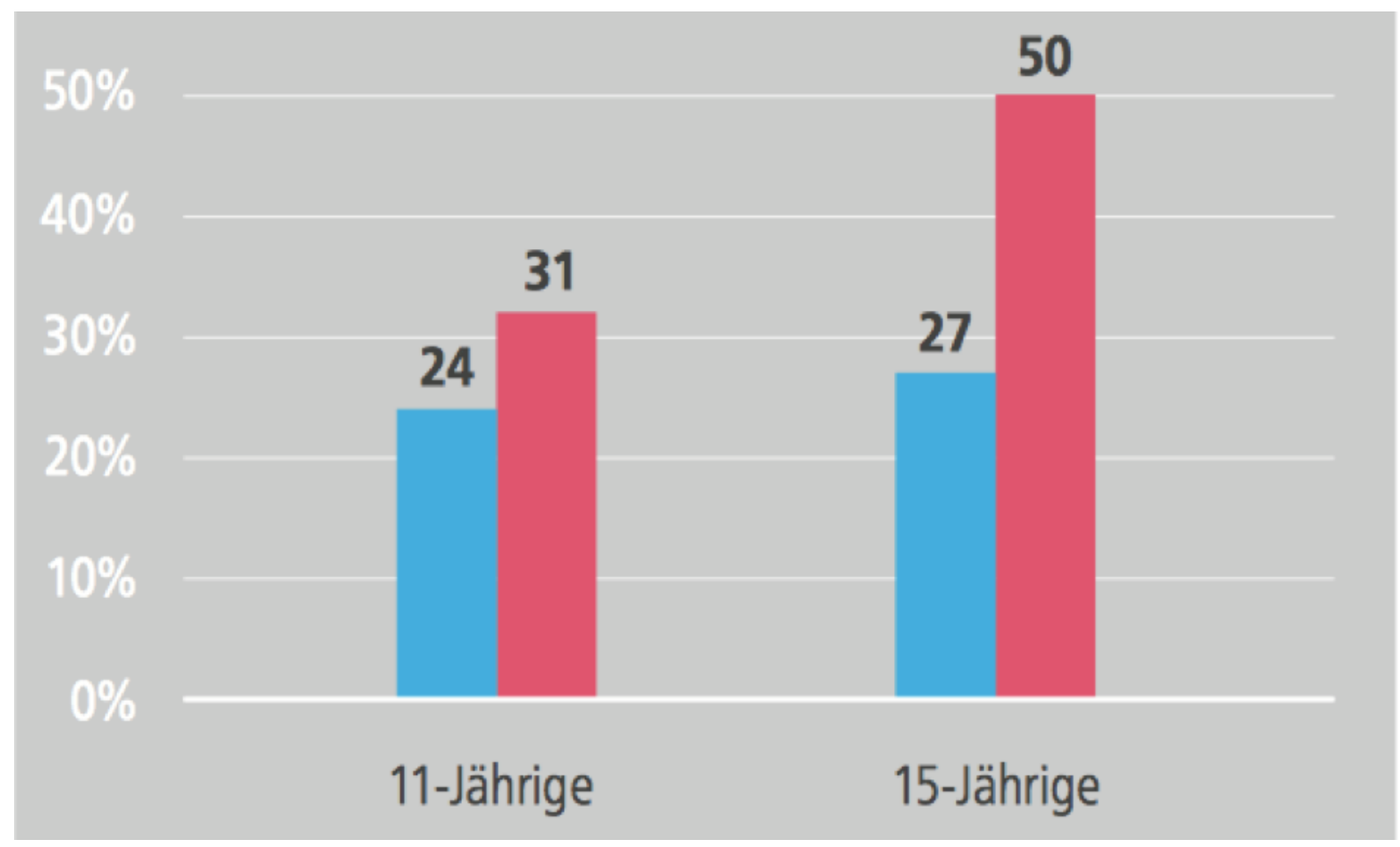

\section{Abbildung 5 hier}




\section{Equity}

Health behaviors in 11,13 , and 15 year-olds, by family affluence

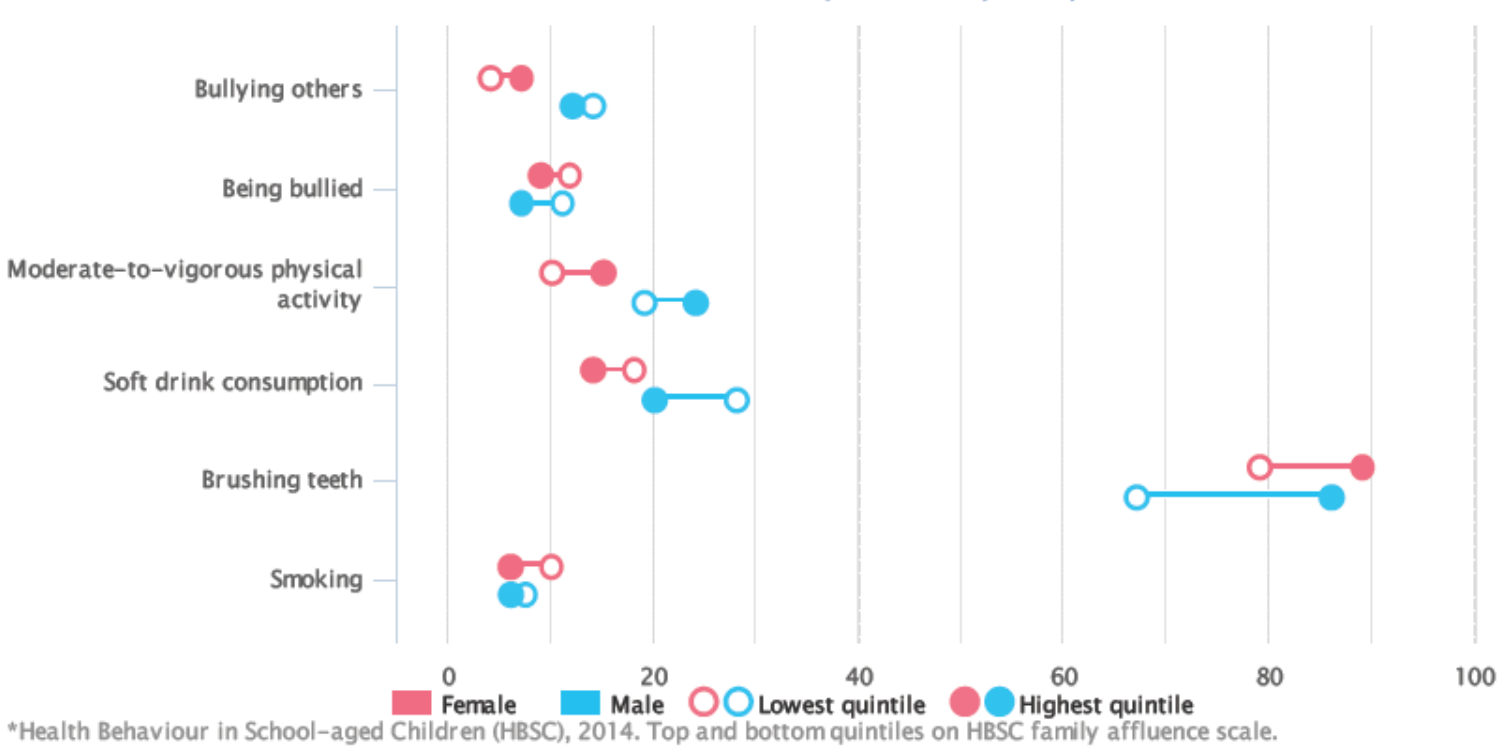

Textbox 2 hier

\section{Kinderpsychiater}

Die Basiserhebung 2016 zeigte, dass es deutliche Unterschiede in der Anzahl der Kinderpsychiater pro 100.000 Kinder zwischen den Ländern der Europäischen Region der WHO gibt: von einem Höchststand von 101 in Estland bis zu nur einem in Tadschikistan und Turkmenistan. Der Median der Kinderpsychiater pro 100.000 Kinder lag bei 6 in den 36 Ländern, die die entsprechenden Informationen darlegten. Die EU15 Länder hatten dabei signifikant höhere Raten an Kinderpsychiatern als Nachfolgestaaten der ehemaligen Sowjetunion (CIS Länder) (Abbildung 6). Daten der Basiserhebung zeigten außerdem, dass die Anzahl von Kinderpsychologen pro 100.000 Kinderpopulation von 172 in Finnland zu zwei in Rumänien reicht. Diese Daten und Vergleiche sind für Länder im Rahmen der Gesundheitsbedarfsplanung wichtig, um einen eventuellen Mangel oder Überversorgung $\mathrm{zu}$ dokumentieren und gegebenenfalls zu korrigieren. Der europäische Vergleich gibt dabei eine Perspektive.

\section{Abbildung 6 hier}




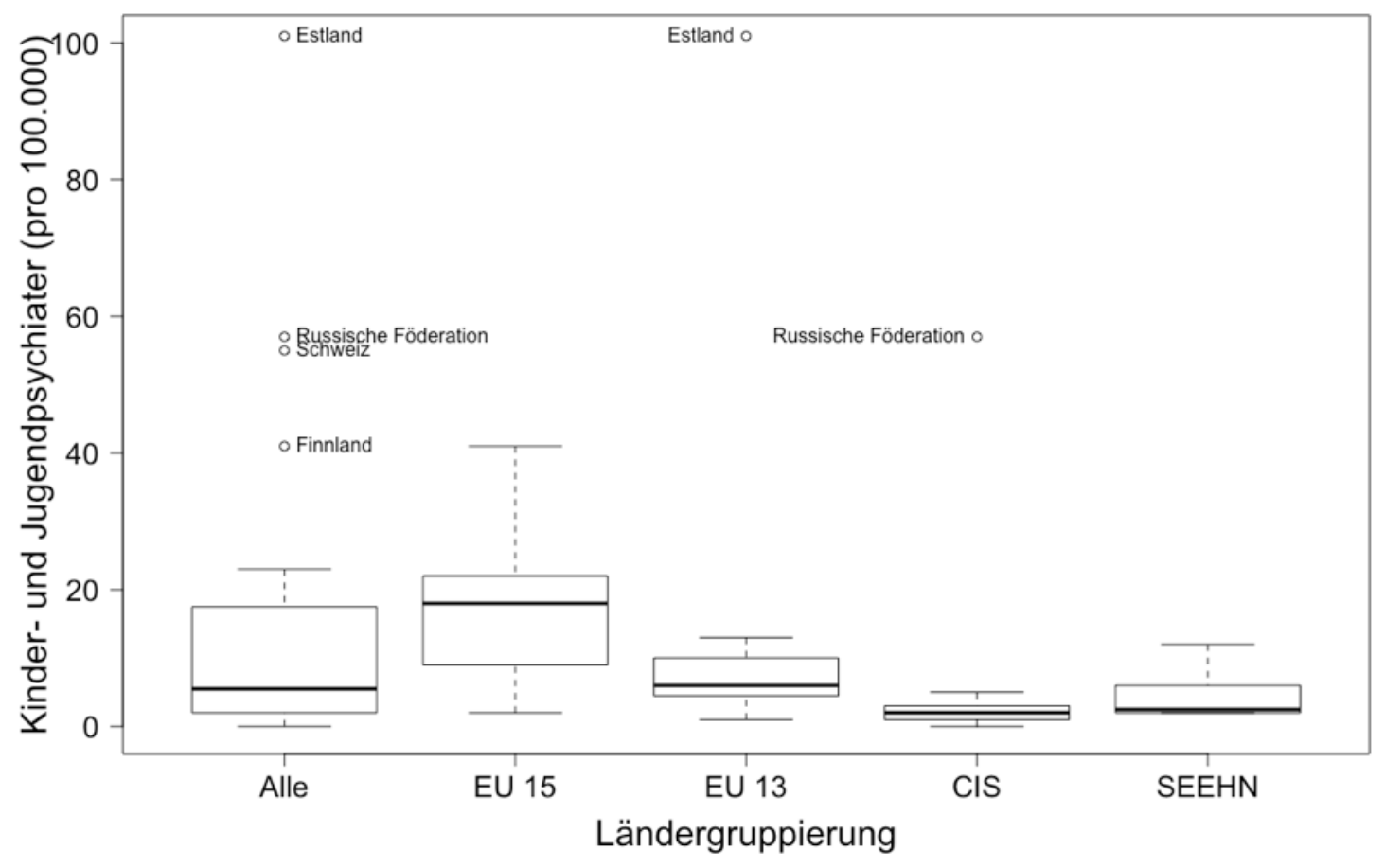

Die Erhebung verweist auf die Notwendigkeit, die psychische Versorgung von Kindern und Jugendlichen in Europa zu verbessern. Die Kinder- und Jugendjahre werden im Europäischen Aktionsplan für psychische Gesundheit [24] als eine kritische Lebensphase beschrieben, die Einfluss auf die psychische Gesundheit im späteren Leben haben. Maßnahmen in diesem Abschnitt sind notwendig, um das psychische Wohlbefinden langfristig über den Lebensverlauf zu verbessern. Das gilt es umzusetzen.

\section{Aspekte der sexuellen Gesundheit bei Jugendlichen}

Sexualität von Jugendlichen ist in manchen Ländern ein Tabuthema, und ihr Zugang zu Dienstleistungen noch viel mehr. In Deutschland gibt es seit 50 Jahren bundesweit einheitliche Empfehlungen für die Sexualerziehung von Jugendlichen [8], die den Sexualkundeunterricht fest im Kurrikulum der Schulen verankerten. Die Bundeszentrale für gesundheitliche Aufklärung, ein Kollaborationszentrum des WHO Regionalbüros, hat europäische Standards zur Sexualerziehung erarbeitet [20]. Laut unserer Befragung werden diese jedoch in den Mitgliedsstaaten inkonsistent und teilweise mangelhaft umgesetzt. So wird der Zugang zu Kontrazeptiva in der Europäischen Region sehr unterschiedlich geregelt. Ein besonders kontroverses Thema ist der Zugang zu Abtreibungen bei Jugendlichen, wie im Folgenden näher beschrieben wird. 
Legaler Schwangerschaftsabbruch ohne elterliche Zustimmung für Jugendliche unter 18 Jahren

Lediglich $48 \%$ der befragten Länder in der Europäischen Region gaben an, dass Jugendliche unter 18 Jahren auch ohne elterliche Zustimmung legal einen Schwangerschaftsabbruch vornehmen lassen können (siehe Abbildung 7). Länder, die mit „Ja” antworteten, beschrieben verschiedene Modelle des Umgangs mit Schwangerschaftsabbrüchen bei Schwangeren unter 18 Jahren, die im Folgenden auszugsweise dargestellt werden.

In Österreich ist ein Schwangerschaftsabbruch ohne die Zustimmung eines Erziehungsberechtigten ab 14 Jahren möglich. Ab diesem Alter wird Schwangeren das Recht eines persönlichen Einverständnisses zur Abtreibung zugesprochen, das nur die Schwangere selbst innehat, da sie als entscheidungsfähig gilt. 14-Jährige, die nicht entscheidungsfähig sind (z.B. aufgrund von Entwicklungsverzögerungen) benötigen die Zustimmung eines Erziehungsberechtigten.

In Großbritannien (England) ist ein Schwangerschaftsabbruch ohne Zustimmung eines Erziehungsberechtigten ab 16 Jahren möglich. Jugendliche unter 16 Jahren können ebenfalls eine vertrauliche ärztliche Beratung, Untersuchung und Behandlung, die auch Abtreibung beinhaltet, in Anspruch nehmen. Mediziner sind verpflichtet, die Reife der Schwangeren in Hinblick auf Gespräche über Einverständnis und Ausbeutung festzustellen. Als optimales Vorgehen gilt, Jugendliche unter 16 Jahren, dringend zum Einbezug der Eltern zu ermutigen. Wird dies abgelehnt, sollte mit Unterstützung des Gesundheitspersonals ein anderer Erwachsener (z.B. ein Familienmitglied oder ein spezialisierter Sozialarbeiter) hinzugezogen werden.

In Ungarn werden Jugendliche zwischen 16 und 18 Jahren als volljährig betrachtet, wenn sie verheiratet sind und haben Zugang zu medizinischen Dienstleistungen, ohne dass das elterliche Einverständnis erforderlich ist. Angaben zu unverheirateten Schwangeren gingen nicht deutlich aus den Ergebnissen der Befragung hervor.

In Dänemark braucht eine nicht verheiratete Person unter 18 Jahren die Zustimmung eines gesetzlichen Vormundes, um einen Schwangerschaftsabbruch durchführen zu lassen. In Ausnahmen darf jedoch eine Schwangere unter 18 Jahren eine Abtreibung auch ohne Einverständnis des gesetzlichen Vormundes in Anspruch nehmen. Eine solche Ausnahme ist 
gegeben, wenn die Gefahr eines Ausstoßes aus der Familie, physischer oder psychologischer Gewalt gegen die Schwangere besteht. Über diese Umstände entscheidet ein Komitee.

\section{Abbildung 7 hier}

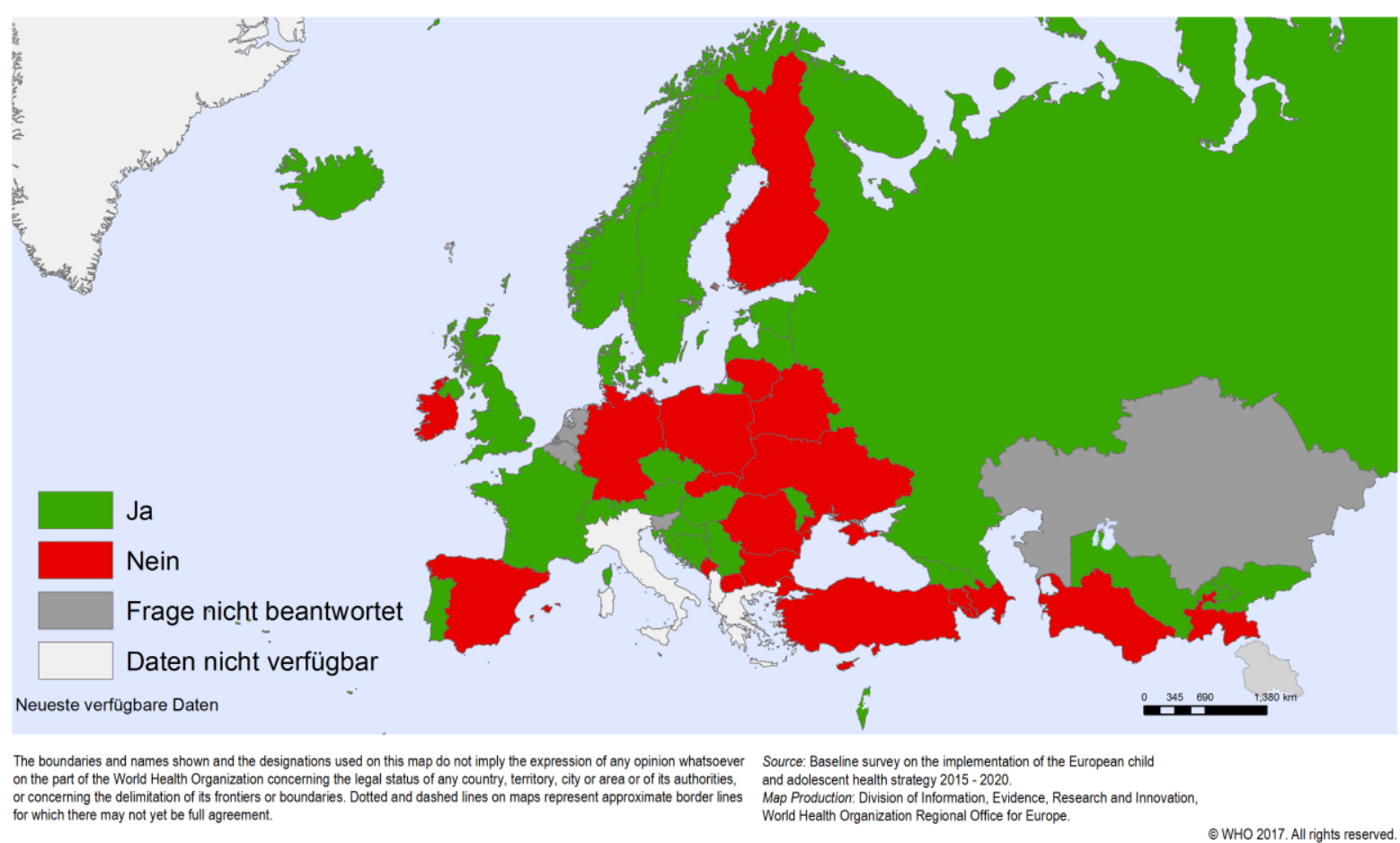

\section{Diskussion und Ausblick}

In diesem Artikel wurden einige zentrale Datenquellen und empirische Erkenntnisse zu Systemen der Kinder- und Jugendgesundheit vorgestellt, die Vergleiche der europäischen Länder zulassen. Diese Vergleiche sind nützlich im nationalen und internationalen Diskurs.

Diesbezüglich ist es allerdings auch wichtig, auf Grenzen der hier beschriebenen Ergebnisse hinzuweisen. So beruhen die Antworten der Basiserhebung auf Auskünften der jeweiligen Gesundheitsministerien. Diese sind unterschiedlicher Qualität und manchmal möglicherweise nicht völlig zutreffend. Außerdem können ausgelassene Antworten sowohl auf fehlendes Verständnis der Frage, mangelnde Informationen als auch auf gewolltes Auslassen zurückzuführen sein. Beispielsweise machten 15 der 48 Länder keine Angaben dazu, ob sie 
Informationen zum Wissen Jugendlicher über Sexualität erheben, was die Interpretation erschwert.

Die durch die Befragung erstellten Länderprofile (link geben zum deutschen Profil) sind damit zunächst als Diskussionsgrundlage für den Dialog mit dem jeweiligen Land zu verstehen. Die WHO befürwortet, dass diese von der Allgemeinheit, und den Kinderärzten im Besonderen, benutzt werden. Weitere Details zu den dichotomen Antworten sind ebenfalls im Fließtext verfügbar, den die WHO auf ihrer Webseite verfügbar machen wird. Die Umsetzung der Strategie wird bis 2020 mit weiteren Erhebungen nachverfolgt, welche auch Indikatoren der in den letzten Jahren beschlossenen nachhaltigen Entwicklungszielen [18] und der globalen Strategie zur Frauen-, Kinder- und Jugendgesundheit [23] einschließen. Auf diese Weise kann nachverfolgt werden, was die Länder unternehmen, und wie sie ihre globalen Verpflichtungen erfüllen. All dies liegt im Interesse der Kinder und Jugendlichen und ihrer Gesundheit.

\section{FAZIT FÜR DIE PRAXIS}

- Die Kinder- und Jugendstrategie der WHO gibt europäischen Ländern eine Anleitung, ihre eigene Herangehensweise für Kinder- und Jugendgesundheit zu entwickeln.

- Der Basiserhebung zur Kinder- und Jugendstrategie erlaubt es Ländern, Themen zu identifizieren, die gestärkt werden müssen, um Gesundheit und Wohlbefinden von Kindern und Jugendlichen zu stärken und sich mit anderen Ländern zu vergleichen.

- Die WHO ermutigt alle Länder, aktiv Daten über verschiedene Bereiche der Kinder- und Jugendgesundheit zu sammeln, und sie für gesundheitspolitische Entscheidungen zu nutzen.

- Nach wie vor mangelt es insbesondere in westeuropäischen Ländern an essentiellen Medikamentenlisten und Darreichungsformen für Kinder und Jugendliche.

- Die WHO stellt den Ländern ein Monitoring-Rahmenwerk auf europäischer Ebene zur Verfügung.

- Pädiater spielen eine wichtige Rolle als Anwälte für die Belange der Kinder, und sollten dafür diese Datenquellen und internationalen Richtlinien kennen. 


\section{Textboxen:}

\section{Textbox 1:}

\section{Prioritäten der Kinder- und Jugendstrategie der WHO für Europa}

1. Leben der Kinder sichtbar machen

2. unerledigte Probleme von vermeidbarer Mortalität und Infektionskrankheiten angehen

3. Regierungshandeln für Kinder- und Jugendgesundheit transformieren
a) Unterstützung von frühkindlicher Entwicklung
b) Unterstützung des Wachstums in Adoleszenz
c) Reduzierung von Gewalterfahrungen

4. Gesundheit schützen und Risiken vermindern
a) Erreichen einer tabakfreien Generation
b) Förderung von gesunder Ernährung und körperlicher Aktivität
c) Bewältigung von Depressionen und anderen psychischen Problemen im Jugendalter
d) Schutz von Kindern und Jugendlichen vor Umweltrisiken

\section{Textbox 2:}

\section{Von der Forschung zu politischen Maßnahmen in der Praxis:}

Ergebnisse der Health Behaviour in School-Aged Children (HBSC) Studie zeigten eine beunruhigende Entwicklung bei deutschen Jugendlichen mit zunehmendem Konsum von Alkopops im Jahr 2003. Die deutsche Regierung reagierte auf die Ergebnisse mit erhöhten Steuern auf Alkopop-Getränke, und Aufklärungskampagenen. Bereits drei Jahre später konnte HBSC deutlich gesunkene Zahlen an jugendlichen Alkopop-Konsumenten in Deutschland verzeichnen.

\section{Textbox 3:}




\section{Wie erfolgt das Feedback an die Mitgliedsstaaten?}

Das Feedback an die einzelnen Länder wurde auf Grund der Basiserhebung 2016 erstellt. Dabei werden den Ländern Errungenschaften im Rahmen der Kinder- und Jugendstrategie 2020 sowie die Bereiche mit Raum für Verbesserungen rückgemeldet. Die Landkarten der Europäischen Region dienen zur Veranschaulichung wie ein Land im europäischen Vergleich abschneidet.

\section{Textbox 4:}

weiterführende Links:

http://www.euro.who.int/en/health-topics/Life-stages/child-and-adolescenthealth/news/news/2017/07/turning-hbsc-study-data-into- action-taxing-alcopops-in-germany https://gateway.euro.who.int/en/country-profiles/germany/\#cah_2 http://www.euo.who.int/_data/assets/pdf_file/0018/314532/66wd13e_SRHActionPlan_160524. pdf

http://www.who.int/medicines/publications/essentialmedicines/6th_EMLc2017_FINAL_amende dAug2017.pdf?ua=1

http://hbsc-germany.de

http://www.hbsc.org

\section{Country-Feedback Germany für Online-Appendix}

https://drive.google.com/open?id=0B28twg0g_Qj5UzJ2bHRqSnM4SUE

\section{Abbildungen}

Abb. 1 Kindersterblichkeit unter 5 Jahren. Dargestellt sind Daten, die die Länder selbst an die WHO berichten (Health for all database) und nach Schätzungen der Weltgesundheitsorganisation. Für einige Länder zeigen sich deutliche Unterschiede.

Abb. 2 Modelle der primärärztlichen Versorgung für Kinder- und Jugendliche in der Europäischen Region 
Abb. 3 Verfügbarkeit einer pädiatrischen essentiellen Medikamentenliste; geografische Verteilung der Antworten aus der Basisbefragung 2016.

Abb. 4 Multiple Gesundheitsbeschwerden einmal pro Woche. Ergebnisse aus der Befragung Health Behaviour in School-aged Children (HBSC) aus dem Jahr 2014

Abb. 5 ausgewählte Risikofaktoren deutscher Jugendlicher aus der Health Behaviours of Schoolage Children Studie, entsprechend den Quintilen der familiären Wohlstandsskala (entnommen von gateway.euro.who.int)

Abb. 6 Anzahl der Kinder- und Jugendpsychiater in den Ländern Europas, nach Ländergruppen (Zugehörigkeit der Länder zu den Gruppierungen siehe Abschnitt "Die Basiserhebung zur Kinder- und Jugendstrategie in den europäischen Ländern”)

Abb. 7 Legaler Zugang zu Abtreibungen ohne elterliches Einverständnis für Jugendliche unter 18 Jahren; geografische Verteilung der Antworten aus der Basisbefragung 2016. 


\section{Literatur:}

1. Bali D, Kuli-Lito G, Ceka N et al. (2016) Maternal and Child Health Care Services in Albania. The Journal of Pediatrics 177:S11-S20

2. Baranov A, Namazova-Baranova L, Albitskiy V et al. (2016) The Russian Child Health Care System. The Journal of Pediatrics 177:S148-S155

3. Bogdanović R, Lozanović D, Pejović Milovančević M et al. (2016) The Child Health Care System of Serbia. The Journal of Pediatrics 177:S156-S172

4. Costello EJ, Mustillo S, Erkanli A et al. (2003) Prevalence and development of psychiatric disorders in childhood and adolescence. Archives of general psychiatry $60: 837-844$

5. Cullu F, Vural M (2016) An Overview on Child Health Care in Turkey. The Journal of Pediatrics 177:S213-S216

6. Jenni OG, Sennhauser FH (2016) Child Health Care in Switzerland. The Journal of Pediatrics 177:S203-S212

7. Kessler RC, Berglund P, Demler O et al. (2005) Lifetime prevalence and age-of-onset distributions of DSM-IV disorders in the National Comorbidity Survey Replication. Archives of general psychiatry 62:593-602

8. Kultusministerkonferenz (1968) Empfehlungen zur Sexualerziehung in den Schulen: Beschluss der Kultusministerkonferenz vom 03.10.1968.

9. López CC (2011) Primary health care in Spain and Catalonia: a nursing model perspective. Revista da Escola de Enfermagem da USP 45:1722-1730

10. Margvelashvili L, Karseladze R, Abesadze G et al. (2016) Child Health Care in Georgia. The Journal of Pediatrics 177:S68-S70

11. Mathiesen P, Maarbjerg SF, Lykke K et al. (2016) The Child Health System in Denmark: Current Problems and Successes. The Journal of Pediatrics 177:S60-S62

12. Models of Child Health Appraised (MOCHA), a study of primary healthcare in 30 European countries. http://www.childhealthservicemodels.eu/. Zugegriffen: 11.11.2017

13. Sargsyan S, Movsesyan Y, Melkumova M et al. (2016) Child and Adolescent Health in Armenia: Experiences and Learned Lessons. The Journal of Pediatrics 177:S21-S34 
14. Tasher D, Rubin L, Grossman Z et al. (2016) Child Health Care in Israel. The Journal of Pediatrics 177:S107-S115

15. Tasic V, Danilovski D, Gucev Z (2016) The Child Health Care System of Macedonia. The Journal of Pediatrics 177:S127-S137

16. Health behaviours of school-aged children. http://www.hbsc.org/. Zugegriffen: 29.10.2017

17. UN General Assembly (1989) The Convention on the Rights of the Child. New York: United Nations. https://treaties.un.org/Pages/ViewDetails.aspx?src=TREATY\&mtdsg_no=IV11\&chapter=4\&lang=en. Zugegriffen: 26.10.2017

18. United Nations (2015) Sustainable development goals. https://sustainabledevelopment.un.org. Zugegriffen: 30. Juni 2015

19. Wolfe I, Sigfrid L, Chanchlani N et al. (2016) Child Health Systems in the United Kingdom (England). The Journal of Pediatrics 177:S217-S242

20. WHO-Regionalbüro für Europa und BZgA (2011) Standards für die Sexualaufklärung in Europa. Rahmenkonzept für politische Entscheidungsträger, Bildungseinrichtungen, Gesundheitsbehörden, Expertinnen und Experten. BZgA, Köln: 41-54.

21. World Health Assembly, Better medicines for children, 23 May 2007, World Health Organization. http://www.who.int/childmedicines/publications/WHA6020.pdf. Zugegriffen: 26.10.2017

22. World Health Organization (2014) Investing in children: The European child and adolescent health strategy 2015-2020. WHO Regional Office for Europe, Copenhagen. http://www.euro.who.int/de/health-topics/Life-stages/child-and-adolescenthealth/policy/investing-in-children-the-european-child-and-adolescent-health-strategy20152020. Zugegriffen: 25.10.2017

23. World Health Organization (2015) Global Strategy for Women's, Children's and Adolescents' Health 2016-2030. Geneva: WHO. http://www.who.int/lifecourse/partners/global-strategy/global-strategy-2016-2030/en/. Zugegriffen: 29.10.2017

24. World Health Organization (2015) The European Mental Health Action Plan 2013-2020. WHO Regional Office for Europe, Copenhagen. 
http://www.euro.who.int/_data/assets/pdf_file/0020/280604/WHO-Europe-MentalHealth-Acion-Plan-2013-2020.pdf. Zugegriffen: 03.11.2017 